\title{
MODELOS DE PARTÍCULAS EN LA INICIACIÓN A LAS CIENCIAS FISICAS
}

\author{
MEHEUT, M., LARCHER, C. y CHOMAT, A. \\ LIRESPT-INRP-Université Paris 7. \\ Versión de Juan Hernández
}

\section{SUMMARY}

First notions about structure of matter are taught in various curricula at the beginning of secondary school. Is it possible with these notions to build explicative and predictive models? What activities can we offer to the students in order to build and use models? We present here two examples of pedagogical approaches and the main directions we have chosen for teaching experiment with students, aged 13-14.

\begin{abstract}
Algunos modelos de particulas son introducidos desde los primeros años de la enseñanza de las ciencias físicas en numerosos curriculos. Trabajos de evaluación de estas enseñanzas han permitido conocer algunas dificultades encontradas por los alumnos en la asimilación de tales modelos.

Los procedimientos pedagógicos propuestos en estos currículos son diversos; presentamos dos aproximaciones muy contrastadas. El primero está centrado en la actividad experimental del alumno y tiene como objetivo la construccion inductiva de un modelo de partículas para explicar los fenómenos estudiados. El segundo aparece más dogmático y consiste, principalmente, en una presentación de modelos más complejos y poco funcionales.

Intentaremos establecer las limitaciones de estos dos tipos de formas de proceder y situarnos en una perspectiva de elaboración y de validación de un modelo elemental de partículas, poniendo en evidencia sus aspectos funcionales.
\end{abstract}

\section{PUNTO DE VISTA EPISTEMOLÓGICO}

La noción de modelo y los diversos aspectos de las actividades de modelización, sea en fases de elaboración, de utilización o bien de validación de un modelo, son temas de numerosos debates en la comunidad científi$\mathrm{ca}$; un punto que nos parece central en este debate es el carácter a la vez figurativo y teórico de un modelo «ambigüedad que se mantiene en todas las variedades del concepto y que está ligada al carácter concretoabstracto de su función" (Bachelard, 1979).

\subsection{Carácter figurativo de los modelos}

Si bien ha sido uno de los fundamentos del desarrollo de la noción de modelo a principios del siglo XIX, este carácter figurativo se atenúa cuando se pasa de los modelos materiales a los simbólicos (matemáticos en particular). Si se considera un sistema y su modelo como dos sistemas homomorfos, el carácter material del modelo se traduce en partículas por la existencia de propiedades formales, propiedades propias al modelo y sin correspondencia en el sistema considerado (Walliser, 1977).

\subsection{Carácter teórico del modelo}

Uno de los aspectos en los que incide este en foque del conocimiento científico es la diversidad de marcos teóricos y su carácter a priori con relación a los sistemas que se desean modelizar, a los fenómenos que se desean simular.

Así, por ejemplo, ciertas transformaciones de un sistema gaseoso pueden describirse por un modelo algebraico constituido por una relación entre magnitudes medibles (presión-volumen-temperatura) o por un modelo cinético de partículas. Este modelo algebraico ha sido considerado en alguna ocasión como empírico, por oposición a una interpretación en el modelo cinético, considerado entonces teórico (Carnap, 1973). Esta oposición empírico-teórico nos parece que debe, sin em- 
bargo, ser matizada. En efecto ningún modelo puede considerarse como desprovisto de toda teoría; por otra parte, el carácter teórico de un modelo evoluciona a lo largo del tiempo:

"Cualquiera que sea el modelo teórico que se considere, siempre se puede intentar poner en evidencia niveles explicativos más profundos de los cuales se le podria hacer derivar... Todo modelo, de un nivel explicativo dado, tiende, por su simple vulgarización y su refuerzo por la experiencia, a aparecer como casidescriptivo al cabo de un cierto tiempo". (Walliser, 1977).

La existencia de varios niveles de explicación supone reglas de correspondencia entre los diferentes niveles. Estas reglas se les llama a veces diccionario, por analogía con las reglas de traducción entre lenguas naturales. Esta analogia ha sido utilizada en particular por Perrin: «Yo protesto rápidamente contra las opiniones que condenan en bloque las teorías moleculares... Podemos proponernos imaginar ciertos modelos, mecánicos, eléctricos u otros, bastante flexibles para ser captados en su conjunto por nuestra razón y, sin embargo, semejantes al universo en cuanto que, al menos a grandes rasgos, sus propiedades y las del universo se corresponden como se corresponderian las frases escritas en dos lenguas diferentes", (citado por Bachelard, 1979).

Uno de los aspectos de la vatidación de un modelo consiste en el establecimiento progresivo de estas correspondencias entre el modelo a validar y los modelos ya aceptados. Estas reglas de correspondencia no permiten sin embargo establecer un isomorfismo entre diferentes modelos de un mismo sistema. En particular, sigue siendo imposible definir totalmente una entidad correspondiente a un nivel explicativo dado en términos más empíricos.

\subsection{Dominio de validez}

La validez de un modelo depende de su coherencia interna y de su simplicidad, pero también, de su poder explicativo y predictivo.

La explicación consiste en describir de manera nueva los fenómenos ya observados, ya descritos por los modelos empíricos. Un modelo es tanto mejor cuanto más extenso sea su dominio de validez, es decir que permite explicar un conjunto más amplio de fenómenos. Esta cuestión de la extensión del dominio de validez se encuentra en las cualidades de previsión del modelo, es decir, en la posibilidad de traducción de simulaciones en fenómenos nuevos. Es en esta confrontación entre simulación y experimentación donde se plantea el problema de la refutación del modelo.

\subsection{Modelos y realidad}

Uno de los aspectos subrayados por la noción de modelo es pues la pluralidad de los modos de descripción que operan en el conocimiento científico.

Esta pluralidad implica la existencia de reglas de correspondencia entre estos diferentes modos de descripción.

El carácter siempre limitado del conjunto de los fenómenos explicables en el marco de un modelo dado (dominio de validez) es otro aspecto que subraya esta aproximación.

Este punto de vista se opone pues netamente a un realismo ingenuo «que tiende, à toda costa, a mantener una identidad entre la realidad y la ciencia, entre la naturaleza y la razón (Bartholy, 1978).

Se opone igualmente a un empirismo radical que «oculta completamente el papel de la hipótesis y de la información teórica» (Bartholy, 1978).

Este punto de vista deja abierto el debate entre realistas científicos y positivistas sobre la existencia de una realidad objetiva, independiente del observador. Todo lo más conduce a afirmar que, si esta realidad existe, el conocimiento cientifico no constituye más que una aproximación progresiva.

\section{PUNTO DE VISTA PEDAGógICO}

\subsection{Análisis de dos formas de enseñanza}

El análisis se refiere a manuales franceses (clase de $4 \dot{e}$ de los collèges) y escoceses (Science for the 70's-Science 2000). Está centrado en:

- el dominio de referencia y su descripción empírica: ¿qué fenómenos se estudian? ¿Cómo son descritos previamente a la introducción de un modelo determinado?

- Ios modelos presentados: su coherencia interna, su poder explicativo y predictivo en el dominio de referencia elegido.

- las actividades propuestas a los alumnos, su lugar en el aprendizaje del modelo.

\subsubsection{En los «collèges» franceses}

E) dominio de referencia no está claramente delimitado: «Toda materia está constituida a partir de átomos» (MEN, 1979). Algunos fenómenos que ponen en juego metales son estudiados más particularmente (incompresibilidad, conducción, reacciones de oxidaciónreducción, electrolisis). El nivel de descripción de estos fenómenos es cualitativo (la corriente pasa/no pasa; el cobre desaparece/reaparece).

Varios modelos se presentan simultáneamente: modelo de bolas duras, modelo de Bohr, de Rutherford, de Sommerfeld, modelo probabilistico (a veces superpuesto sobre una misma imagen).

El léxico es rico: átomo, núcleo, electrón, ión, a veces moléculas, protón, neutrón. 
El acento está puesto sobre los aspectos figurativos de los modelos: «La utilización de imágenes simples y concretas deberá ser siempre buscado» (MEN, 1979). De hecho las representaciones utilizadas son muy variadas no solamente de un manual a otro, sino también de un capitulo a otro dentro de un mismo manual.

Si estos modelos son complejos en lo que concierne a] léxico y los aspectos figurativos, son pobres en lo que concierne a las reglas de funcionamiento del modelo mismo (¿cuáles son las «historias) posibles para los átomos, los electrones...?) y sus aspectos funcionales. Aparecen muy parcialmente explicativos del comportamiento de los sistemas modelizados, incluso inadaptados. (Figura 1).

La complejidad de los modelos presentados es tal que resulta difícil hacerlos funcionar (para que el modelo explique los fenómenos de oxidación-reducción por ejemplos, falta añadir aspectos energéticos, que están aqui totalmente ausentes). De hecho, ninguna actividad de utilización del modelo está propuesta a los alumnos. La problemática no es hacer funcionar un modelo, tomar la medida de su poder explicativo o predictivo, de sus reglas de funcionamiento, sino describirlo tal como es:

\section{«1. Realidad del átomo.}

En nuestros días, la realidad de estas parcelas, llamadas átomos, no está puesta en duda por nadie, porque físicos y químicos han acumulado un gran número de pruebas experimentales de su existencia» (Chirouze, 1979).

Este enfoque oculta, de una parte, los aspectos teóri* cos de los modelos presentados y, de otra parte, las re. glas de correspondencia que fundamentan su validez. Ello nos parece contradictorio con una iniciación a la metodologia científica, $y$ nos aproxima más a la ideologia y al dogmatismo:

"La ciencia es totalmente consciente de este valor instrumental de los modelos, ella no confunde la verdad significada con los contenidos limitativos de la figuración, evita así el dogmatismo que resultará de una confusión del objeto con sus modelos" (Mouloud, 1985).

Parece que esta presentación no-funcional de determinados modelos no sea especifico ni de la enseñanza francesa ni del nivel de enseñanza que hemos considerado aquí; en particular, una comprobación análoga ha podido hacerse al llevar a cabo el andilisis de obras para la enseñanza secundaria y universitaria en España:

«La introducción del modelo cuántico... es en general incorrecto y confuso porque: se trata de una introducción lineal y sin estructurar en las cuales se yuxtaponen simplemente (o se mezclan) las concepciones clásicas (modelos atómicos de Thomson, Rutherford), con las antiguas teorias de los cuantos (modelos de Bohr, Sommerfeld), y las concepciones cuánticas (Schrodinger, Heisenberg)" (Solbes et al. 1987).

\subsubsection{En el "Scottish Integrated Science Course»}

En las ediciones 1970, el procedimiento pedagógico que debe seguirse está bastante explicitado; el acento está puesto sobre la actividad experimental de los alumnos y sobre el papel inductor de esta actividad:

«La naturaleza de la materia: Los alumnos realizan experiencias a partir de las cuales llegarán a la conclusión de que la mejor explicación de sus observaciones es que la materia está formada por partículas) (Mee, 1971).

Se trata de una didáctica del descubrimiento:

«Objetivos específicos: Capacidad de utilizar un razonamiento inductivo para construir una teoría de la es. tructura de la materia a partir de observaciones experimentales" (Mee, 1971).

Se proponen numerosas experiencias. Algunas relativas a las nociones de presión de un gas, de masa específica. Otras se refieren a fenómenos físicos (dilatación) o químicos.

Los términos de la observación de estos fenómenos no están nada explicitados. Esto es totalmente coherente con el punto de vista empirista adoptado, que oculta los aspectos teóricos de la observación y «supone que la observación (la sensación) es el punto de partida del conocimiento» (Bartholy, 1978).

En la perspectiva de descubrimiento adoptada, ningún modelo se presenta a los alumnos. Los modelos contemplados son cinéticos y descansan sobre un léxico limitado: átomo y molécula.

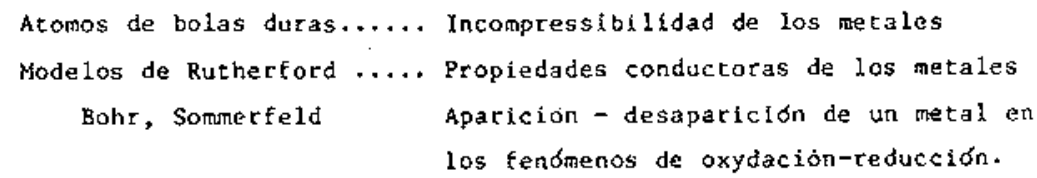


La evaluación de esta enseñanza ha conducido a una puesta en cuestión de este procedimiento didáctico, ya que la actividad experimental de los alumnos no les conduce en absoluto a elaborar modelos de partículas en la materia (Mitchell, 1982).

\subsection{Investigaciones recientes en este dominio}

Esta perspectiva de adoptar un modelo determinado por la observación de fenómenos «bien escogidos» es el origen de diferentes investigaciones.

Puede citarse, a este respecto, un estudio de Pfundt (1981): se trata de entrevistas efectuadas a varios alumnos de 12 a 14 años (49 alumnos); los fenómenos estudiados son la evaporación y la condensación de agua, la disolución de cristales de sulfato de cobre y la evaporación de la disolución obtenida. En el transcurso de estas entrevistas, los alumnos desarrollan concepciones continuistas de la materia. Los fenómenos escogidos para suscitar el conflicto cognitivo es aquí la comprensibilidad de los gases opuestos a la incompre. sibilidad de los líquidos.

Teniendo en cuenta las condiciones contradictorias de los diferentes trabajos llevados a cabo desde esta perspectiva, nosotros hemos retenido, en una fase exploratoria, algunas situaciones citadas en la literatura como susceptibles de permitir la presentación, léase la inducción del carácter discreto de la materia como respuesta a preguntas sobre la existencia de un límite a la divisibilidad. Estas entrevistas y cuestionarios escritos nos han mostrado que estos fenómenos no suscitan entre los alumnos descripciones en términos de discontinuidad de la materia; las propiedades macroscópicas les resultan suficientes para explicar estos fenómenos.

Los resultados de esta fase exploratoria (a publicar) nos conducen a pensar que los fenómenos considerados en un gran número de currículos como inductores del mo. delo discreto de la materia no desempeñan en absolu. to este papel. Nos encontramos aquí de nuevo con los límites de una epistemologia empirista cuyas implicaciones didácticas han sido objeto de discusiones recientes (Gil 1986, Llorens 1987, en particular).

\section{EXPERIMENTACIÓN DIDÁCTICA: IN- TENTO DE APRENDIZAJE DE PROCEDI- MIENTOS DE MODELIZACIÓN}

Se trata de una experimentación «en el medio natural» puesto que se desarrolla en la propia aula de colegios de la región parisina, dentro del horario escolar y en clases no excepcionales.

Este tipo de experimentación presenta dificultades: dificultades temporales para la puesta a punto y la repetición de las experiencias, dificultades relativas a la elección de los sistemas estudiados (aquí las clases de $4^{\circ}$ en condiciones «normales», disponen de un material «normal». Sin embargo, permite un ajuste pragmático de las perspectivas, preguntas, proposiciones, etc. a las posibilidades cognitivas de los alumnos y a las cuestiones de funcionamiento de la institución escolar.

La muestra sobre la que se realiza el estudio está cons. tituida por 14 clases de $4^{\circ}$ de colegio de la región parisina (alrededor de 400 alumnos). Esta muestra no se ha elegido en función de su representatividad sino de la motivación de los enseñantes: las caracteristicas socio-culturales de los colegios elegidos son, sin embargo, variados.

\subsection{Concepción de las sesiones de aprendizaje}

En la concepción de estas sesiones, hemos tratado de poner a los alumnos en situación de desarrollar las actividades que nos parecían esenciales en la actividad cientifica conocidas como elaboración y utilización de modelos

Los sistemas modelizados han sido muestras de materia geseosa, de sólidos y líquidos; los fenómenos estudiados en el curso de las sesiones fueron transforma ciones físicas tales como la compresión de un gas, la mezcla por difusión de dos gases, el cambio de estado sólido-gas. La interpretación de otros fenómenos físicos: (difusión de un gas a través de una pared sólida, dilatación de un sólido o de un liquido.... disolución de un sólido, fusión, evaporación) ha sido objeto de un cuestionario escrito al finalizar estas sesiones de aprendizaje.

La modelización necesita una descripción empírica previa de los fenómenos (observación) y la elección de un cuadro teórico para la interpretación de estos fenóme. nos. Así, para cada fenómeno estudiado, una primera etapa ha consistido en que los alumnos efectúen una descripción del mismo. Sólo ciertos términos de esta descripción serán retenidos para dar lugar a más modelización.

Con el fin de salir al paso de una tendencia espontánea de los alumnos a atribuir a las partículas las propiedades observables de la materia (Bain 1984, Driver 1983, Meheut, 1982), nosotros hemos preferido in troducir a priori el carácter elemental de estas partículas: indivisibilidad, indeformabilidad y masa invariante. Los aspectos de invarianza son en efecto esenciales para que una descripción de la materia basada en partículas pueda adquirir un carácter explicativo y predictivo (Halbwachs 1973, O'Neil 1972).

En una segunda etapa, los alumnos han sido invitados a interpretar algunas de sus observaciones utilizando un modo de representación icónica.

Los modelos propuestos por los alumnos han sido sometidos a discusion, en una tercera etapa; esta discusión ha versado, por una parte, sobre la no contradicción entre estas representaciones y el carácter elemen- 
tal de las partículas; por otra parte, sobre los aspectos significativos o no de los modelos así producidos.

\subsection{Técnicas de observación}

A lo largo de las sesiones de aprendizaje, los escritos de las actividades de los alumnos (descripción empírica de los fenómenos, producción de un modelo, discusión de los modelos propuestos) han sido recogidos en todas las clases.

En tres clases, las intervenciones de los profesores, las discusiones de clase y las discusiones de varios grupos de alumnos han sido registrados en magnetófono.

\subsection{Análisis de los datos recogidos}

Presentamos a continuación de forma detailada los datos recogidos durante la modelización de un fenómeno: el de la compresión de un gas. Los datos recogidos en el momento de la modelización de los otros fenómenos estudiados serán presentados más sucintamente.

\subsubsection{Interpretación de la compresión de un gas}

3.3.1.1. Descripción empirica del fenómenoobservación.

El enseñante comprime un gas coloreado (dióxido de nitrógeno) en una jeringa y dibuja en la pizarra la posición del pistón en dos instantes diferentes.

Pide a los alumnos que realicen individualmente y por escrito una descripción del fenómeno. (Figura 2).

La forma de la pregunta tiene por objeto conducir a una descripción en términos de cambio de estado de la muestra de gas encerrado en la jeringa, ya que cada estado está caracterizado por un conjunto de valores de las variables que describen el sistema «muestra de gas"s, estos valores pueden sufrir o no una variación en el momento de la transformación estudiada.

Resultados: La compresión del gas fue descrita por un tercio de los alumnos en términos de invariancia de la cantidad de gas $(36 \%)$, por otros en términos de inva* riancia de la masa $(12 \%)$, el color $(25 \%)$ y la naturaleza del gas $(10 \%)$ son igualmente evocados. El cambio está descrito por una elevada proporción de alumnos $(60 \%)$ en términos de paso a un estado de «apisonamiento». Las otras variaciones evocadas son principal- mente las del volumen del gas $(36 \%)$ y de su color $(39 \%)$.

Para describir este fenómeno, los alumnos utilizan pues magnitudes físicas tales como la masa y el volumen y descripciones cualitativas tales como el color, el "apisonamiento"). Puede observarse que la presión ha sido poco evocada, a pesar de que ha sido estudiada en clase de $6^{\circ}$. Esto nos parece que se debe a dos elementos de la didáctica utilizada: de una parte la manipulación ha sido efectuada por el enseriante, lo que ha suprimido toda percepción directa de las acciones mecánicas por los alumnos, de otra parte la cuestión estaba planteada en términos de descripción de estados de una muestra de gas lo que ha podido eliminar ciertos elementos de descripción de lo sucedido.

\subsubsection{Traducción de las observaciones. Producción de un modelo.}

Entre los elementos de descripción propuestos por los alumnos algunos son recogidos por el enseñante; se trata de la invariancia de la cantidad y de la naturaleza del gas por una parte, y de la variación del volumen y del estado de «apisonamiento» por la otra.

El enseñante propone entonces representar la muestra de gas como un conjunto de objetos elementales (partículas) e interpretar el cambio de estado del gas en esta nueva descripción. (Figura 3).

Los cuadros propuestos para la representación de la muestra de gas son escogidos de forma que representen la variación del volumen ocupado por la muestra en el curso de la compresión. Los alumnos no tienden a traducir en este término la observación.

Resultados: Una gran mayoria de los alumnos ha aceptado la idea de utilizar este nuevo lenguaje; en efecto el $84 \%$ de entre ellos han trazado para las dos situaciones, símbolos idénticos traduciendo el «apisonamiento" del gas mediante un acercamiento de estos simbolos gráficos.

Una escasa proporción de entre ellos (23\%) conservó el mismo número de símbolos; la conservación de la cantidad no fue pues más que poco interpretada; este hecho nos parece que puede ser explicado por la im. precisión de la consigna dada a los alumnos («Representad el gas...») o por la incomprensión del significa-

figura 2

\begin{tabular}{|l|l|l|}
\hline De la situación 1 & Para la jeringa & Para el gas \\
a la situación 2 & & \\
\hline Que es lo que ha cambiado? & & \\
\hline Qué es lo que no ha cambiado? & & \\
\hline
\end{tabular}


figura 3

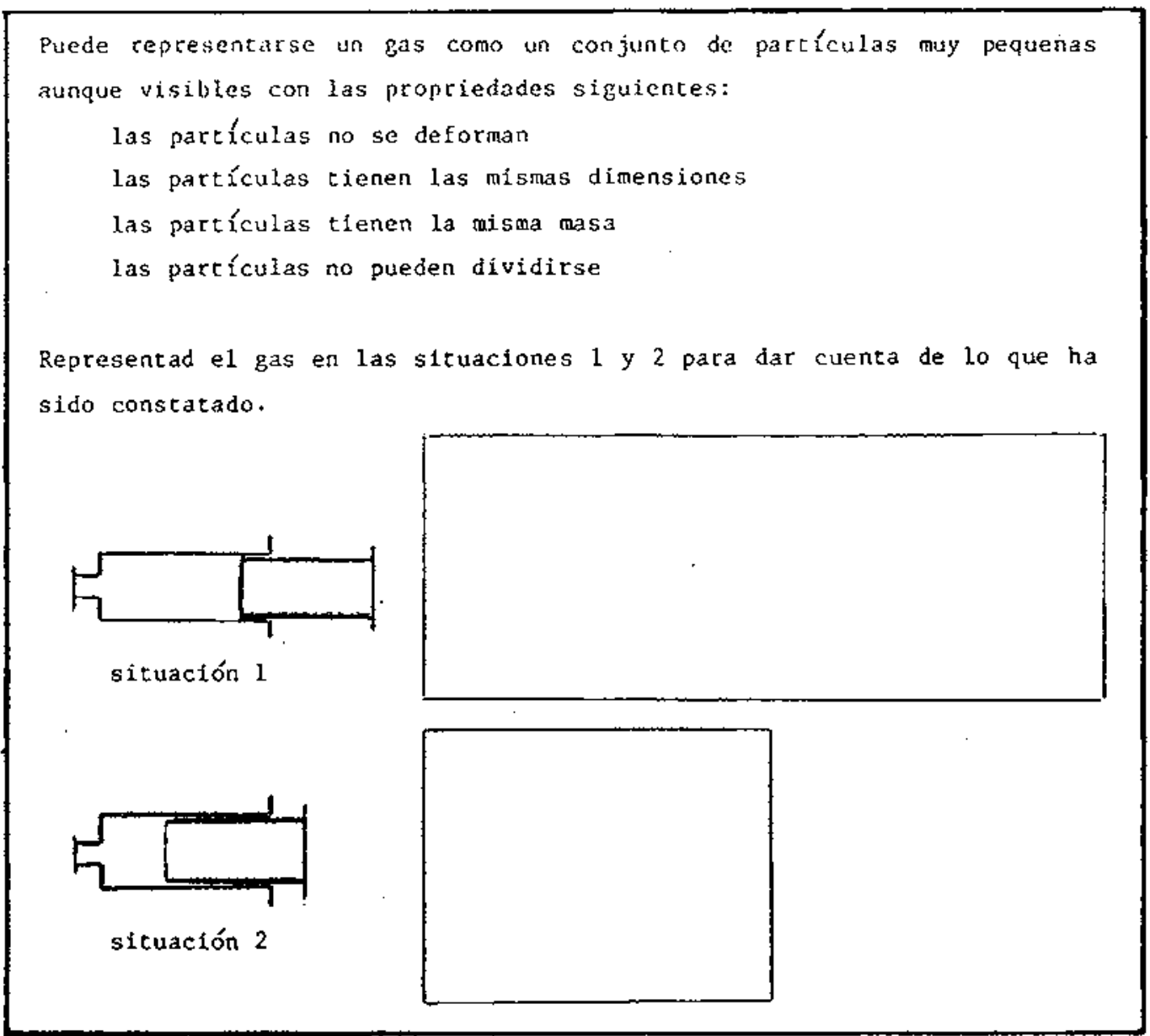

do de los marcos propuestos. Por esta razón, hemos modificado las preguntas en 1987. Esta modificación persigue hacer participar al alumno en la elección del marco y convertir en más explícita la tarea solicitada.

\subsubsection{Crítica de los modelos producidos}

Tres alumnos reproducen en el cuadro las representaciones que han propuesto. La coherencia de estas representaciones con la descripción macroscópica previamente efectuada es discutida en pequeños grupos; el resultado de la discusión es objeto de un escrito individual.

Resultados: Aproximadamente la mitad de los alumnos explicitan las relaciones. (Fígura 4).

Pero un alumno de cada cuatro, aproximadamente, no hace más que comentar la validez de la descripción empírica:

— «si se pesan los dos tubos, se encuentra el mismo peson

figura 4

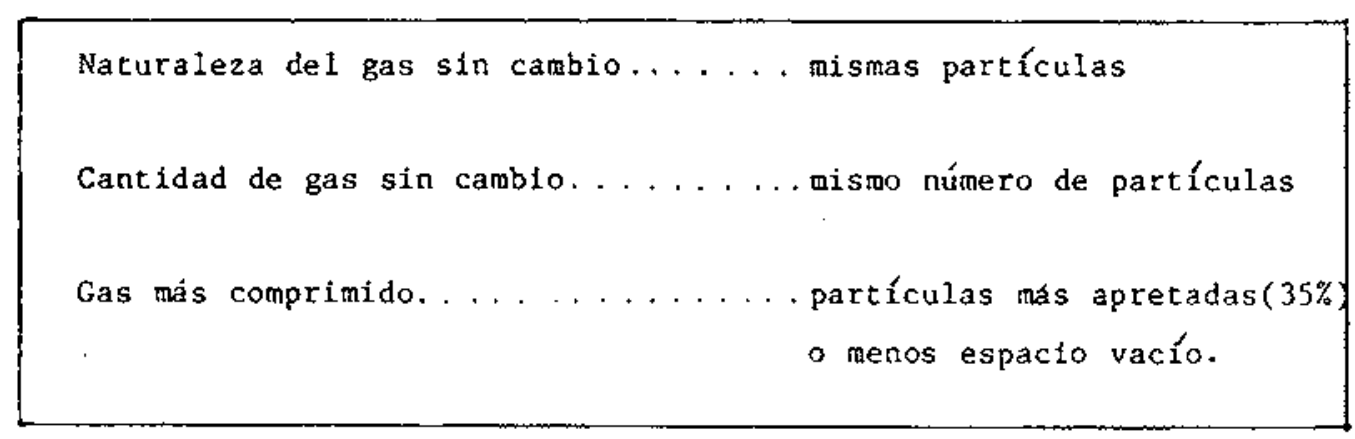


- «no se ha quitado o aportado gas»

- «si, no se ha añadido gas, sólo se le ha comprimido».

\subsubsection{Aplicación del campo de referencia y evolución del modelo}

Una segunda secuencia de enseñanza versó sobre la interpretación de una mezcla por difusión de dos gases. A continuación se interpretaron algunas propiedades de la materia, en estado sólido, líquido, gaseoso (posibilidad de comprensión y deformación, cohesión (y un cambio de estado sólido-gas).

Resultados: La interpretación de la mezcla de dos gases condujo a un alumno de cada cuatro, aproximadamente, $23 \%$, a introducir aspectos cinéticos:

«Las partículas deben tener la posibilidad de poder mezclarse, asi pues, de desplazarse».

«Las partículas se reparten igualmente en las dos botellas, asi pues, se mueven».

«Las partículas pueden desplazarse a cualquier lugar (en cualquier sentido".

Por lo que se refiere a la modelización de un sólido, aproximadamente la mitad de los alumnos $(52 \%)$ proponen un modelo estático. Son poco numerosos (12\%) los que mantienen una cierta agitación. Un 50\% de ellos afirman que en un sólido las particulas están un poco espaciadas, siendo el argumento que proporcionan el de la débil compresibilidad de los sólidos; el 17\%, sin embargo, consideran el contacto entre partículas.
"No hay vacío puesto que las partículas están ligadas". «El espacio entre las partículas no está vacio, las partículas no tienen ninguna distancia entre ellas, están ligadas y pegadas".

\subsection{Conclusiones}

En el transcurso de estas sesiones, los alumnos han:

- propuesto interpretaciones de algunos fenómenos físicos en el marco de modelos de partículas.

- discutido en pequeños grupos las reglas de correspondencia entre descripción empírica de estos fenómenos y modelos propuestos.

- discutido la coherencia interna de los modelos propuestos.

Los modelos construidos por los alumnos no son, por supuesto, modelos cinéticos estadísticos; su poder explicativo se refiere principalmente a la conservación de la materia (conservación de la masa-conservación de la sustancia), a pesar de que se ha modificado su estado perceptiblemente en varios fenómenos físicos (compresiones, dilataciones, cambios de estado, mezclas).

Nota: En Francia los cursos correspondientes a $6,7,8$ y 1 de BUP se cursan en los «collèges». De 1 de EGB a 5 de EGB, se cursan en la Escuela Primaria (Ecole Primaire). En el Insituto (Lycée) se cursan 2, 3 de BUP y COU, que en Francia se denominan respectivamente Deuxième, Première y Terminale (COU).

\section{REFERENCLAS BIBLIOGRÁFICAS}

BACHELARD, S., 1979, Quelques aspects historiques des notions de modèle et de justification des modèles, Actes du colloque: Elaboration et justification des modeles, 1 , 3.19.

BAIN, D., BERTRAND, F, 1984, Structure de la matière: des représentations des éléves aux représentations des manuels, Signes et discours dans l'éducation et la vulgarisation scientifique, 441-448. (UER de didactique des disciplines, Université Paris 7).
BARTHOLY, M.C., DESPIN, J.P., GRANDPIERRE, G., 1978, La science, épistémologie générale. (Magnard: Paris).

BROOK, A., BRIGGS, H., DRIVER, R., 1984, Aspects of secondary students' understanding of the particulate nature of matter, The University of Leeds.

CARNAP, R., 1973, Les fondements philosophiques de la physique. (Colin: Paris). 
CHIROUZE, P.J. y al, 1979, Sciences physiques, classe de 4ème. (A. Colin: Paris).

GIL, D., 1986, La metodología científica y la enseñanza de las ciencias. Unas relaciones controvertidas. Enseñanza de las ciencias, 4 (2), 111-121.

HALBWACHS, F., 1973, Histoire de l'explication en physique, $L$ 'explication dans les sciences, $72-\$ 19$. (Flammarion, Paris).

LLORENS, I.A., 1987, Tesis doctoral, Universidad de Vaiencia.

MEE, A.J., BOYD, P., RITCHIE, D., 1971, Science for the 70's, Teacher's guide I. (Heinemann Educational Books Ltd.: London).

M.E.N., 1979, Sciences Physiques, Programme et Instructions pour la clasșe de 4ème, Bulletin officiel, $\mathrm{n}^{\circ}$ spécial 4 bis du 11.1.79, Paris.

MITCHELL, A.C., NOVICK, S., 1982, Learning difficulties associated with the particulate theory of matter in the
Scottish Integrated Science Course, European Journal of Science Education, 4, 429-440.

MOULOUD, N., 1985, Modele: perspective épistémologique, Encyclopedia Universalis, Vol 12, 412.415.

NUSSBAUM, J., NOVICK, S., 1982, Alternative frameworks, conceptual conflict and accomodation: toward a principled teaching strategy. Intructional Science, 11, 183-200.

PFUNDT, H., 1981, The final link in the division process or the firsty building block? Pre-instructional conceptions about the structure of substances. Chimica Didactica, 7, $75-94$.

SOLBES, J., CALATAYUD, M.L., CLEMENT, J.B., NAVARRO, J., 1987, El modelo cuántico del átomo. (Universitat de València: València).

WALLISER, B., 1977, Systèmes et modèles, (Ed. du Seuil, Paris). 